\title{
Benchmarking the dental randomised controlled literature on MEDLINE
}

Richard Niederman, ${ }^{1}$ Lisa Chen, ${ }^{2}$ Linda Murzyn $^{2}$ and Suzy Conway ${ }^{3}$

${ }^{1}$ Forsyth Institute; ${ }^{2}$ Harvard School of Dental Medicine; and ${ }^{3}$ Countway Medical Library, Harvard Medical School, Boston, Massachusetts, USA

Objective To develop and implement MEDLINE search strategies that access the dental literature in each of seven dental disciplines; to estimate the availability of randomised controlled trials (RCT) in these disciplines that might be used to make clinical decisions; and to examine publication trends.

Study design Search strategies based on Medical Subject Headings (MeSH) for endodontics, implant dentistry, oral surgery, oral medicine/radiology, orthodontics, periodontics and restorative dentistry were developed to examine MEDLINE. Publications were limited to studies in humans, the years 1990 to 2000, and multiple methods of ascertainment employed to identify RCT. Ascertainment methods included maximally sensitive, sensitive, MEDLINE

publication type and specific search strategies. Publication trends were examined using one search methodology.

Results In the period 1990-2000, the MEDLINE searches identified a substantial number of RCT that differed significantly from one another, in ascending order by search strategy: specific $(1260)<$ MEDLINE RCT $(2443)<$ sensitive $(6006)<$ maximally sensitive $(26808)[\mathrm{P}<0.001$ in analysis of variance (ANOVA)]. The number of RCT varied significantly between the dental disciplines. For example, using the MEDLINE RCT, in ascending order, the number of RCT were: implant dentistry $(104)<$ endodontics $(106)<$ orthodontics $(198)<$ restorative dentistry $(420)<$ oral surgery $(438)<$ periodontics $(983)<$ oral medicine/radiology (984) ( $\mathrm{P}<0.001$, ANOVA). Trends over the 10year period indicate that the number of RCT is increasing substantially in the aggregate for dentistry, and for each dental discipline. The range is approximately 10-20\% per year, depending on the search strategy.

Conclusions The results indicate that, (1) there is a substantial clinical literature of RCT in MEDLINE for clinical and curricular decision making; (2) the increasing number of RCT suggests the need for computer-based clinical knowledge systems; and (3) the methods used here can be employed to identify the most current clinical RCT literature in each of the dental disciplines. The results, however, require two caveats. First, the search results vary significantly, based on the search strategy. Thus, the results can be expected to 'bracket' the real number. Second, a truer measure of the clinical utility of each citation will be achieved only by individually assessing the validity and clinical applicability of each article. Within these limitations, these findings provide an estimate of the availability of the dental discipline specific therapeutic literature, and have implications for educational, clinical, research and policy decision makers.

Evidence-Based Dentistry (2002) 3, 5-9. DOI: 10.1038/sj/ ebd/6400095

Please note that the appendix tables for this paper are to be found on the journal's website

\section{Introduction}

To identify and verify the availability of clinical information in the dental literature for evidence-based clinical decision-making, we conducted a benchmarking study of MEDLINE. The reason for this study is that the increasing emphasis on the development of evidence-based clinical decision-making ${ }^{1-4}$ places more focus on the availability of high quality evidence. $^{5,6}$ Further, access to computer-based communication networks and, online, criticallyappraised medical information can potentially improve clinical decisionmaking by increasing information availability. ${ }^{7,8}$

Evidence-based clinical decisionmaking requires the presence of and access to a large volume of high- quality clinical information. Without such literature, evidence-based clinical decision making would be impaired.

We employed bibliometric methods (the analysis of a literature using statistical methods to reveal the historical development of subjects and publications $)^{9}$, methods successfully used for similar purposes in medicine. Bibliometric methods are currently being used by the United States National Academy of Sciences for evaluating research programs ${ }^{10}$ and have been used for evaluating clinical progress in cardiology, ${ }^{11}$ audiology, ${ }^{12}$ mental health, ${ }^{13}$ epilepsy, ${ }^{14}$ emergency medicine, ${ }^{15}$ family practice, ${ }^{16}$ allied health, ${ }^{17}$ arthritis ${ }^{18}$ and dentistry. ${ }^{4,19,20}$

The objectives of the current study were to develop and implement
MEDLINE search strategies that would access the dental literature, then estimate the availability of RCT that could potentially be used to make clinical and curricular decisions, and to examine trends. This study in some ways parallels and expands on recent work examining the diagnosis and management of dental caries $^{21}$. This is a first step toward developing computer-based clinical knowledge systems to assist in clinical and curricular decision analysis.

\section{Methods}

Literature search: Seven dental disciplines were selected for the study: endodontics, implant dentistry, oral surgery, oral medicine/radiology, orthodontics, periodontics and 
restorative dentistry. For each discipline a MEDLINE search strategy was developed (see Appendix, Tables la-7a) using dental vocabulary identified from the MEDLINE Medical Subject Heading (MeSH) index ${ }^{22}$ (see Appendix, Tables 1b-7b). Literature searches were performed using the Ovid Web Gateway (Ovid Technologies, New York, New York, USA) internet interface for MEDLINE (see http://gateway.ovid.com). Initially, MEDLINE was searched from 1966 to February (week 4) 2001. The identified literature was limited to studies in humans and to the years 1990-2000 (inclusive). The identified literature was then further limited to MEDLINE 'Publication Type' to "randomised controlled clinical trials". RCT in particular were selected because they are thought to represent the highest level of therapeutic evidence $6,23,24$ (http://cebm. jr2.ac.uk/docs/levels.html). Next, three alternative search strategies were implemented to identify RCT. These validated strategies were obtained from Clinical Evidence ${ }^{26}$ (http:// www.clinicalevidence.org/what-is-ce/ search-strategy-appraisal-page-4.htm). The first of the three was a maximally sensitive search, while the latter two searches were of decreasing sensitivity and increasing specificity (see Appendix, Tables $8 \mathrm{a}-\mathrm{c})$.

Calculations: To estimate the total number of RCT, a capture-recapture method was used. ${ }^{27}$ This estimate is made as follows:

$$
N=[(M+1)(n+1) /(m+1)] \quad 1
$$

where $N$ is the estimated number of existing publications, $M$ is the number of publications identified by MEDLINE, $n$ is the number identified by another search strategy and $m$ is the number identified by both sources. The estimated number unidentified by either method was calculated by subtrac- tion. The variance of $N$ is estimated as:

$$
\begin{aligned}
\operatorname{Var}(N)= & {\left[(M+1)(n+1)\left(\begin{array}{ll}
M & m
\end{array}\right)\right.} \\
& \left(\begin{array}{ll}
n & m)
\end{array}\right) /\left[(m+1)^{2}(m+2)\right]
\end{aligned}
$$

where $\operatorname{Var}(N)$ is the standard deviation, $M$ is the number of publications identified by MEDLINE, $n$ is the number identified by another search strategy and $m$ is the number identified by both sources.

To address the occurrence of overlap of the articles identified by more than one discipline, an additional search was run. Conceptually, a single discipline-specific search was employed and the Boolean "not" function was used to combine this search with an inclusive search of all the other disciplines combined. The combined search was developed by using the Boolean "or" function to combine all of the other dental disciplines. The proportion of overlap of the single discipline with other disciplines were calculated as:

[ (number of RCT identified for one discipline number of RCT unique to the discipline)/(number of RCT of all other disciplines combined) $] \times 100$

The mean percentage of the overlap was calculated by averaging the ratios from each of the seven disciplines.

Statistical analysis: The data-sets were analysed using Instat 2.01 (Graphpad Software, San Diego, California, USA). To determine whether the results of the search strategies were similar or statistically different, we examined the ANOVA with Bonferroni's correction for multiple comparisons.

Table 1 Estimated number of RCT in dentistry, 1990-2000

\begin{tabular}{lcccc}
\hline & \multicolumn{4}{c}{ Papers $(\mathrm{n})$} \\
\cline { 2 - 5 } & Medline RCT & Most sensitive & Sensitive & Specific \\
\hline Identified & 2443 & 26808 & 6006 & 1260 \\
Computed & & 26820 & 6009 & 3575 \\
Unidentified & 12 & 3 & 2315 \\
\hline
\end{tabular}

\section{Results}

Each of the search strategies provided different results. The data (Table 1) indicate that the identified number of RCT in dentistry ranges from approximately 1300 to 27000 for the four search strategies. As expected, the greatest number of articles was identified by the maximally sensitive search strategy. This was nearly 21 fold greater than the number of articles identified by the specific search. In ascending order, by search strategy, the total number of RCT identified in dentistry was: specific $(1260)<$ MEDLINE RCT $(2443)<$ sensitive $(6006)<$ maximally sensitive (26 808). The range varied significantly and was indicative of the variability of the search strategies used to identify RCT $(\mathrm{P}<0.001$, ANOVA).

We next examined individual dental disciplines. As in dentistry as a whole, the number of RCT varies considerably between the search strategies and the dental disciplines. The lowest number of identified articles was found for Implant Dentistry (10 to approx. 1600). The greatest number of articles was found in Oral Medicine and Radiology (approximately 600-12 000). The number of RCT by discipline and by search method differed significantly from one another ( $\mathrm{P}<0.001$, Kruskal-Wallis). For example, in order of ascending number of articles identified, the dental disciplines for the MEDLINE RCT search strategy were: implant dentistry $(104)<$ endodontics (106) $<$ orthodontics $(198)<$ restorative dentistry $(420)<$ oral surgery (438) $<$ periodontics $(983)<$ oral medicine/radiology (984).

In Table 1, the number of articles identified in dentistry for each 
Table 2 Estimated total number of RCT in dental disciplines, 1990-2000

\begin{tabular}{|c|c|c|c|c|}
\hline & \multicolumn{4}{|c|}{ Papers (n) } \\
\hline & $\begin{array}{l}\text { MEDLINE } \\
\text { RCT }\end{array}$ & $\begin{array}{c}\text { Most } \\
\text { sensitive }\end{array}$ & Sensitive & Specific \\
\hline \multicolumn{5}{|c|}{ Implant dentistry } \\
\hline Identified & 104 & 1580 & 299 & 8 \\
\hline Computed & & 1596 & 302 & 315 \\
\hline \multicolumn{5}{|l|}{ Endodontics } \\
\hline Identified & 106 & 1514 & 317 & 23 \\
\hline Computed & & 1529 & 321 & 160 \\
\hline \multicolumn{5}{|l|}{ Orthodontics } \\
\hline Identified & 198 & 3364 & 559 & 41 \\
\hline Computed & & 3381 & 562 & 398 \\
\hline \multicolumn{5}{|c|}{ Restorative dentistry } \\
\hline Identified & 420 & 6060 & 1334 & 108 \\
\hline Computed & & 6075 & 1338 & 684 \\
\hline \multicolumn{5}{|l|}{ Oral surgery } \\
\hline Identified & 438 & 3518 & 850 & 308 \\
\hline Computed & & 3527 & 852 & 560 \\
\hline \multicolumn{5}{|l|}{ Periodontics } \\
\hline Identified & 983 & 5946 & 1909 & 578 \\
\hline Computed & & 5953 & 1911 & 1479 \\
\hline \multicolumn{5}{|c|}{ Oralmedicine and radiology } \\
\hline Identified & 984 & 12065 & 2497 & 553 \\
\hline Computed & & 12078 & 2500 & 1443 \\
\hline
\end{tabular}

strategy seems relatively low compared to the number that would result from adding the number of RCT for the individual dental disciplines in Table 2. (eg, 2443 versus 3233 for the MEDLINE RCT totals). This discrepancy suggests an overlap of the articles between the various specialties. To test this hypothesis, we determined the number of articles included in a single discipline that also occurred in other disciplines. The mean proportion of overlap was $42 \%( \pm 15 \%)$ for MEDLINE RCT searches, $44 \%$ ( $\pm 10 \%)$ for maximally sensitive searches, $46 \%( \pm 10 \%)$ for sensitive searches, and 45\% ( $\pm 19 \%)$ for specific searches.

Because the results of the search methods varied so markedly between the four search strategies, a capturerecapture method ${ }^{27}$ was used to estimate the actual number of RCT. The number of articles missed by any method could be calculated by subtraction and verifies (Table 1) that the calculated number of randomised control trials in dentistry ranges from approximately 3575 to 26 820. Once again, the maximally sensitive search strategy identified the greatest number of articles. In ascending order, by search strategy, the total number of RCT estimated in dentistry was specific (3575) < sensitive $(6009)<$ maximally sensitive $(26820)(\mathrm{P}<0.001$, ANOVA $)$. It was observed that the identified and computed numbers of RCT most closely related for the sensitive search strategy (three potentially missed articles) whereas the numbers were least closely related for the specific search strategy (approximately 2300 potentially missed articles).

Finally, linear regression analysis of the number of RCT in dentistry, per year, over a 10 -year period was used to determine a trend line (Figure 1), which illustrates that the number of dental RCT is increasing substantially. The number of articles has doubled over the 10-year period and the rate of change is approximately $10 \%$ per year which is significant $(\mathrm{P}<0.0003$, ANOVA).

\section{Discussion}

The current study was conducted to identify and estimate the availability of RCT literature that could be used to make clinical and curricular decisions. This is a first step toward developing online knowledge systems for clinical decision-making. The different search strategies provided a range of the estimated number of RCT available for the 10-year period 1990-2000. The estimate lies between 1300 (specific search) and 27000 (maximally sensitive search), verifying that there are a substantial number of RCT in dentistry. There is a variation in the search strategies used to identify the RCT because of the significant difference in the estimates derived from each method. The same is true for the dental disciplines (eg, a specific search for implant dentistry yields eight citations compared with 578 for periodontics).

Each of the search strategies, however, have certain limitations. First, certain RCT may have been omitted, whereas other non-RCT may have been included. It was with this issue in mind that the search strategies attempted to bracket the available literature by using sensitive and specific searches. As expected, the maximally sensitive search, in all disciplines, retrieved more articles than a specific search. Second, the classification of articles and their isolation from other specialties was made based on the MEDLINE MeSH headings. As a result, although the word selection was meant to be inclusive, it may have excluded some relevant articles and included a number of irrelevant ones. Third, the data presented here for all searches are likely to be upper estimates of the number of articles that provide valid, clinically applicable information at a high level of evidence. Further assessments are needed to critically appraise the identified articles (see http:// cebm.jr2.ox.ac.uk/docs/downloads.html) and determine the level of evidence for each article prior to implementation (http://cebm.jr2.ox.ac.uk/docs/ levels.html). Because of the large variability among the search methods, the likelihood that we did not identify all of the RCT and the potential for overlap among the dental disciplines, we used additional methods to address this. To more closely estimate the actual number of RCT we 

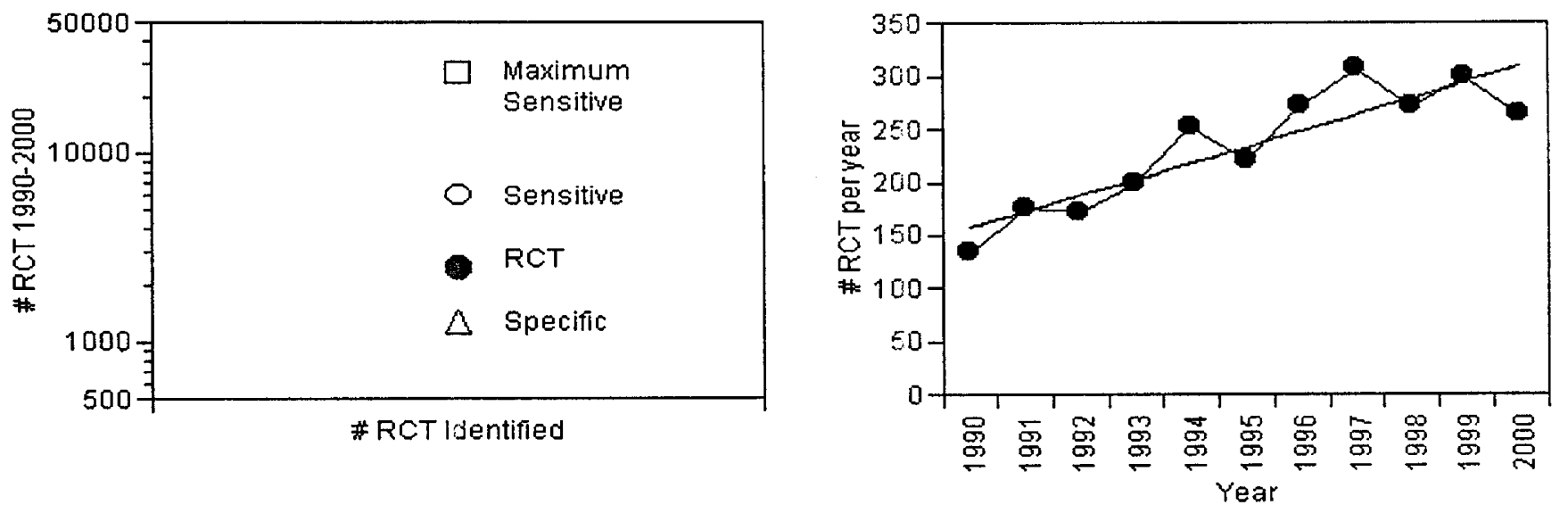

Figure 1 Trend Line of MEDLINE Dental Randomized Controlled Trials 1990 to 2000. (a) Number of RCT identified by four search strategies. Each search strategy identified a substantially different number of randomised controlled trials. (b) Trend Line indicating the number of RCT/year 1990-2000 (linear regression)

employed capture-recapture methods. Although the term capture-recapture may not be as appropriate in literature searches as in ecological determinations of population size, as applied here, the term 'ascertainment intersection' might be used, or the descriptor, 'comparison of multiple methods of ascertainment'. ${ }^{27}$ Independent of the language employed, the concepts are similar. That is they are methods by which the actual size of the population may be determined.

Our trend line analysis (Figure 1) of MEDLINE RCT indicated that there are currently, on average, about 300 RCT per year. As indicated (Table 1), this figure may be reduced by half (for specific searches) or increased 10-fold (for maximally sensitive searches). Using the MEDLINE RCT as a discussion point, the data suggest that to stay current with the literature, academics and clinicians may need to read six articles each week, 52 weeks per year. The trend analysis also indicates that the number of articles is likely to continue to increase. This large and increasing volume of information also begins to suggest a reason for the large variation in clinical care. Variation in health care is chronicled yearly by the Dartmouth Atlas of Healthcare (http://www.dartmouthatlas.org/default.php). Their evidence indicates that extraordinary variation in care and outcomes is associated with three factors, poor quality of science underlying clinical care, poor quality of clinical decision making, and variation in level of clinical skill. These factors may also account, in part, for the recent report in Reader's Digest ${ }^{28}$ highlighting the variability of treatment plans, and for the highly regarded series of scientific studies demonstrating significant variability in dental diagnosis and treatment. ${ }^{29}$ Our data suggest a fourth factor, the large and increasing volume of literature.

These trends suggest the need for computer-based clinical knowledge systems. Thus, the next steps are to sample, critically appraise, stratify and electronically catalogue the identified literature to provide an accessible and ongoing electronic database. Such a database will be useful for multiple audiences. These audiences include academics concerned about the evidence base for curricular decisions; patients and clinicians making decisions about clinical care; researchers interested in identifying gaps in the available knowledge base; corporate entities interested in developing new products; policy makers who fund clinical research; healthcare purchasers who make decisions about care compensation; and finally for professional societies that seek to provide guidance for their membership.
Finally, the search methods employed here can be applied immediately to identify RCT in a given dental discipline. For example, the term "bleaching" can be 'anded' with endodontics to identify RCT of endodontic bleaching (see Appendix, Table $8 c$, line 5). The search results will include a small number of very relevant RCT, but with very few irrelevant articles.

\section{References}

1. Haynes RB, Sackett DL, Gray JA, Cook DJ, Guyatt $\mathrm{GH}$. Transferring evidence from research into practice: overcoming barriers to application. ACP J Club 1997; 126:A1415.

2. Sackett DL, Rosenberg WM, Gray JM, Haynes RB, Richardson WS. Evidence based medicine: what it is and what it isn't. BMJ 1996; 312:71-72.

3. Richards D, Lawrence A. Evidence-based dentistry. Br Dent J 1995; 179:270-273.

4. Niederman R, Badovinac R. Tradition-based care and evidence-based care. J Dent Res 1999; 78:1288-1291.

5. Greenhalgh T. How to read a paper: the MEDLINE database. BMJ 1997; 315:180183.

6. Greenhalgh T. How to read a paper: assessing the methodological quality of published papers. BMJ 1997; 315:305-308.

7. Lindberg DAB, Humphreys BL. Medicine and health on the internet: the good, the bad and the ugly. JAMA 1998; 280:13031304.

8. Bader SA, Braude RM. "Patient informatics": creating new partnerships in medical decision making. Acad Med 1998; 73:408-411. 
9. Young H. ALA Glossary of Library and Information Science. Chicago: American Library Association 1983.

10. Griffiths $P A$, Alberts $B M$, Diamond $P$, et al. Evaluating federal research programs. Washington, DC: National Academy Press 1999.

11. Yosipovitch G, Belhassen B. Bibliometric study of cardiology in Israel in 1978, 1983 and 1988. Isr J Med Sci 1991; 27:234238.

12. Lutman M. Bibliometric analysis as a measure of scientific output. Br J Audiol 1992; 26:233-234.

13. Adams CE, Power A, Frederick K, Lafebvre C. An investigation of the adequacy of MEDLINE searches for randomised controlled trials (RCT) of the effects of mental health care. Psych Med 1994; 24:741-748.

14. Marson AG, Chadwick DW. How easy are randomised controlled trials in epilepsy to find on Medline? The sensitivity and precision of two Medline searches. Epilepsia 1996; 37:377-380.

15. Singer AJ, Homan CS, Stark MJ, Werblud MC, Thode Jr HC, Hollander JE. Comparison of types of research articles published in emergency medicine journals. Acad Emerg Med 1997; 4:1153-1158.

16. Van der Weijden $T$, ljzermans $C J$, Dinant $G$ J, vanDuijn NP, de Vet $R$, Buntinx F. Identifying relevant diagnostic studies in MEDLINE. The diagnostic value of the erythrocyte sedimentation rate (ESR) and dipstick as an example. Fam Pract 1997; 14:204-208.
17. Schloman BF. Mapping the literature of allied health: project overview. Bull Med Libr Assoc 1997; 85:271-277.

18. Lewison G, Devey ME. Bibliometric methods for the evaluation of arthritis research. Rheumatology 1997; 38:13-20.

19. Russo SP, Fiorellini JP, Weber H-P, Niederman R. Benchmarking the dental implant evidence on MEDLINE. Int J Oral Maxillofacial Implants 2000; 15:792-800.

20. Kim MY, White R, Lin J, Niederman R. Benchmarking the endodontic literature on MEDLINE. J Endod 2001 Jul; 27(7):470373.

21. Glock M, Horowitz AM. Diagnosis and Management of Dental Caries. Current Bibliographies in Medicine. Bethesda: US Department of Health and Human Services, Public Health Service, National Institutes of Health, National Library of Medicine 2001 (no. 1).

22. US Department of Health and Human Services. Medical Subject Headings. Bethesda: Public Health Service, National Library of Medicine 1999.

23. US Department of Health and Human Services. Acute Pain Management: Operative or Medical Procedures and Trauma. Rockville: Public Health Service, Agency for Health Care Policy and Research 1992 (AHCPR publication no. 92-0038).
24. Guyatt GH, Sackett DL, Sinclair JC, Hayward R, Cook DJ, Cook RJ. Users' Guides to the Medical Literature. IX. A Method for Grading Health Care Recommendations. Evidence-Based Medicine Working Group. JAMA 1995; 274:1800-1804.

25. Haynes RB, Wilczynski N, McKibbon KA, Walker CJ, Sinclair JC. Developing optimal search strategies for detecting clinically sound studies in MEDLINE. J Am Med Inform Assoc 1994; 1:447-458.

26. Dickersin K, Scherer R, Lefebrve C. Identifying relevant studies for systematic reviews. BMJ 1994; 309:1286-1291.

27. Spoor P, Airey M, Bennett C, Greensill J, Williams R. Use of the capture-recapture technique to evaluate the completeness of systematic literature searches. BMJ 1996; 313:342-343.

28. Echenbarger W. How honest are dentists? Readers Digest 1997 (Feb):50-56.

29. Bader JD, Shugars DA. What do we know about how dentists make caries-related treatment decisions? Comm Dent Oral Epidem 1997; 25:97-103. 